\title{
JD6 - The Connection between Radio Properties and High Energy Emission in AGNs
}

\author{
Gabriele Giovannini $^{1,2}$ and Teddy Cheung ${ }^{3}$ \\ ${ }^{1}$ Department of Physics and Astronomy, Bologna University, Italy \\ email: ggiovann@ira.inaf.it \\ ${ }^{2}$ Istituto di Radioastronomia-INAF, Italy \\ ${ }^{3}$ Naval Research Laboratory, Washington, DC 20375, USA \\ email: Teddy.Cheung@nrl.navy.mil
}

\begin{abstract}
While observations in the radio band are providing essential information on the innermost structures of relativistic jets in active galactic nuclei (AGN), the recent detection by Fermi of gamma-ray emission from many hundreds of blazars shows that the maximum jet power is emitted at high energies. Multi-wavelength monitoring observations further allow variability studies of the AGN spectral energy distributions over 13 orders of magnitude in frequency. The Joint Discussion offered the possibility for a comprehensive discussion of advances in the observational domain and stimulated theoretical discussion about our current understanding of jet physics.
\end{abstract}

Keywords. galaxies: active, BL Lacertae objects: general, galaxies: jets, quasars: general, gamma rays: observations, radio continuum: general

\section{Introduction}

A Joint Discussion (JD) dedicated to the connection between radio properties and high energy emission in active galactic nuclei (AGN) took place on the 23rd and 24th of August 2012 during the XXVIII IAU General Assembly in Beijing, China. It was a great opportunity to confront observational multiwavelength data with theoretical models for AGN. The JD included four main sessions covering the AGN population in the radio and gamma-ray bands $(\S 2)$, high resolution core and jet properties $(\S 3)$, multiwavelength correlations and variability $(\S 4)$, and a final discussion on jet physics and the role of black hole $(\mathrm{BH})$ spin and accretion $(\S 5)$. Eight invited plus 11 contributed oral talks were presented together with 34 contributed posters to an interesting scientific discussion. In the following we present a brief summary for the talks in the four sessions.

All presentations are available at: http://www.ira.inaf .it/meetings/iau2012jd6/ Program.html and poster abstracts in: http://www.ira.inaf.it/meetings/ iau2012jd6/Posters.html.

\section{Session 1: The AGN population as seen in the Radio and Gamma-ray bands}

2.1. T. Cheung - The AGN population in Radio and Gamma-rays: Origins and Present Perspective (invited)

Radio observations present a rich phenomenology in studies of AGN - extensive multifrequency lightcurves (e.g., Fig 1), observations and statistics of superluminal motions, 
radio spectral polarimetric imaging on all scales (jets, hotspots, lobes), and an abundant variety of source types including young radio sources and radio-quiet objects. In the EGRET era, there were 66 high-confidence blazars identified with gamma-ray sources (27 lower-confidence) and only a few radio galaxies (e.g., Cen A, 3C 111). Now we are in the era of the Fermi Gamma-ray Space Telescope with the increased capabilities provided by the Large Area Telescope (LAT). The 2nd LAC AGN catalog (2LAC) "clean" sample included: 310 flat spectrum radio quasars (FSRQs), 395 BL Lacs, 156 blazars of unknown type, and 24 AGNs (include radio galaxies). FSRQs have been detected with $L_{\gamma} \sim 10^{48}$ $\mathrm{erg} / \mathrm{s}$ up to $z \sim 3$. Significant correlations are present between radio and gamma-ray properties. FSRQs are on average brighter and apparently more luminous in the radio band than BL Lacs (but a redshift incompleteness is present).

Thanks to the 1st Fermi-LAT Hard source Catalog (1FHL) which includes data from August 2008 through July 2011, possibly softer AGN gamma-ray spectra appear with increasing redshift, and many new targets are now available for current and future TeV telescopes. The high energy emission site for AGN can be probed with gamma-ray imaging in exceptional cases, like in the giant radio lobes of Centaurus A (and possibly in NGC 6251, and Fornax A). Moreover Fermi detected giant gamma-ray bubbles in our Galaxy $(\S 5.4)$ and a possible young radio source $(4 \mathrm{C}+55.17)$. In the coming years, it will be possible to extend the radio/gamma-ray correlations to low fluxes/luminosities, to continue to identify possible sites of gamma-ray emission, to test if radio-quiet AGN are also gamma-ray quiet, and to look for bubble sources in nearby galaxies.

References: Abdo et al. (2010a), Ackermann et al. (2011b), McConville et al. (2011), Paneque et al. (2012), Takeuchi et al. (2012).

\subsection{E. Stawarz - The AGN population in Radio and Gamma rays: theoretical perspectives (invited)}

Radio-loud systems appear to be the only AGN loud in gamma-rays. In addition to blazars and radio galaxies, the only newly established class of gamma-loud AGN are the radio-loud narrow line Seyfert 1s (NLS1s). Radio-quiet Seyferts seem gamma-quiet as well. Radio properties of nuclear jets may be directly related to gamma-ray properties. However, nuclear relativistic jets are not the only relevant gamma-ray production site as lobes and bubbles are observed in gamma-rays. Correlations between radio and gammaray properties seem to be present, but more data and better statistics are necessary to understand if they are real or not. In flux-flux limited samples, artificial correlations are expected. Evidence of co-spatiality is not firmly established.

References: Abdo et al. (2009b), Ackermann et al. (2012), Ghirlanda et al. (2011), HESS Collaboration (2012)

\subsection{H. Sol - Very high energy gamma-ray radiogalaxies and blazars (contribution)}

At present, 53 firmly known very high energy (VHE; > $0.1 \mathrm{TeV}$ ) AGN have been observed. Among them we have 47 blazars, 4 radio galaxies and 1 AGN of unknown type and possibly Sgr A*. The blazar sample includes: 34 high-frequency peaked BL Lacs (HBL), 4 intermediate-frequency peaked BL Lacs (IBL), 4 low-frequency peaked BL Lacs (LBL), 3 FSRQ, and 2 BL Lacs. The four radio galaxies are: M87, Cen A, NGC 1275, IC 130. Most of them are beamed sources with a strong Doppler boosting as expected since it helps to accommodate fast variability and to avoid strong intrinsic absorption. Variability time scales are from a few minutes to months and years. Multi-zone synchrotron self-Compton (SSC) models can reproduce most of HBL stationary state spectra.

References: Acciari et al. (2011), Aleksić et al. (2012), Abramowski et al. (2012). 


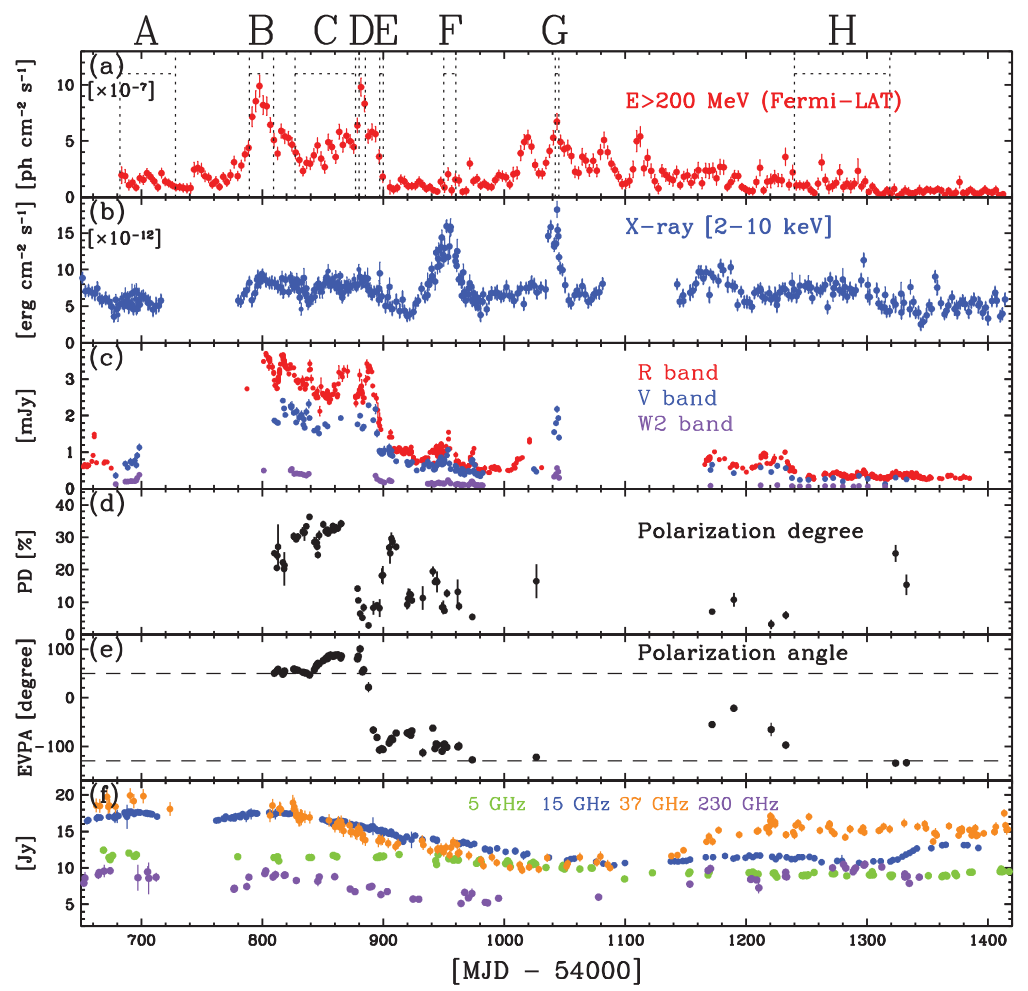

Figure 1. Multi-band (gamma-ray, X-ray, optical, including polarization degree and EVPA, and radio lightcurves of 3C 279 from 2008 August to 2010 August. Taken from Hayashida et al. (2012).

\subsection{McConnell - Counterparts to Fermi-LAT sources from the ATPMN 5 and $8 \mathrm{GHz}$ catalogue of southern radio sources (contribution)}

The Australia Telescope Parkes-MIT-NRAO (ATPMN) catalogue gives accurate positions and flux density measurements at 4.8 and $8.6 \mathrm{GHz}$ for 8385 sources, with a typical image rms noise of $2 \mathrm{mJy} /$ beam. Significant long term variability for $85 \%$ of the sources was detected using the 10-14 year baseline between the ATPMN and AT20G observations. Short term variations were gauged by inspecting ATPMN scans separated by up to 4-8 hrs, with about $30 \%$ showing intra-day variability. Polarization is available for 9040 sources. Among them 126 sources have been identified with 2FGL sources and 112 have an optical identification within $3^{\prime \prime}$ from the UK Schmidt survey.

Reference: McConnell et al. (2012).

\subsection{Karouzos - Gamma-rays in flat-spectrum AGN: revisiting the fast jet hypothesis (contribution)}

Based on studies using EGRET and the early Fermi-LAT AGN samples, gamma-ray detected AGN were found to show on average faster apparent speeds with respect to other AGN not detected in gamma-rays. To confirm and investigate this, the very long baseline interferometry (VLBI) proper motion results for 198 QSOs and 33 BL Lacs from the Caltech Jodrell Bank Flat-spectrum (CJF) 5 GHz VLBI survey were analyzed (Fig. 2). Among these 61 sources have been detected by Fermi-LAT, consisting of 32 FSRQs, 24 BL Lacs, and 5 radio galaxies. Conclusions are: i) no strong link is present between fast jets and gamma-ray detection; ii) AGN class and gamma-ray variability are 


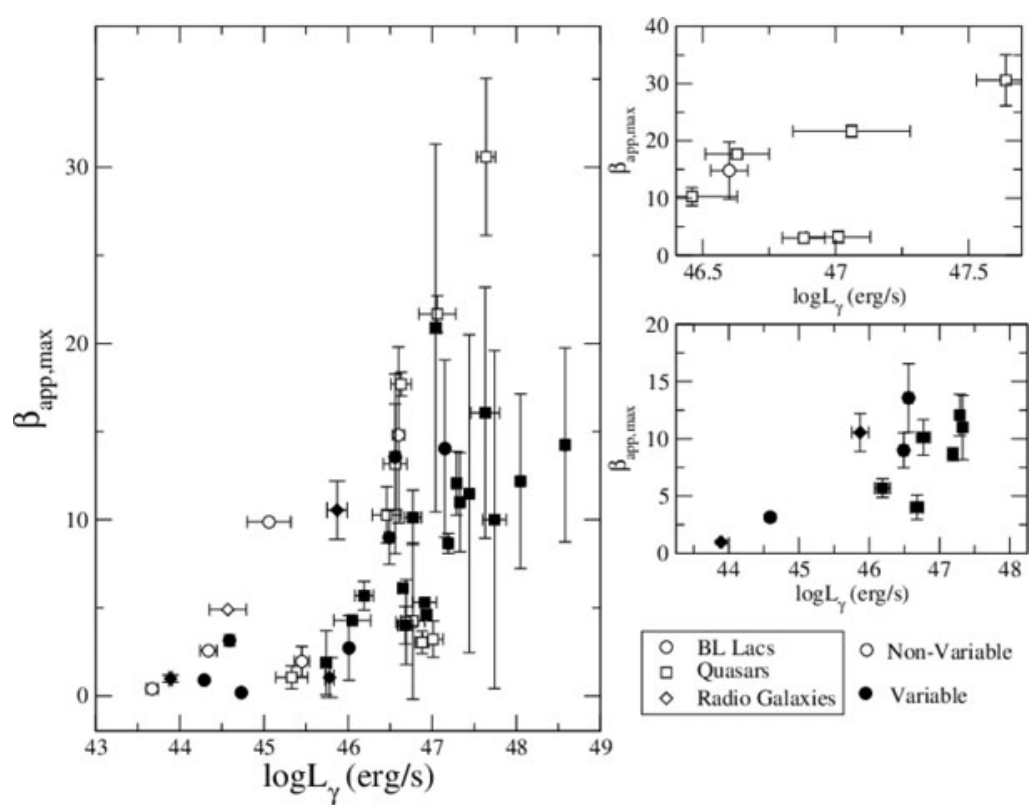

Figure 2. Maximum VLBI measured apparent velocities for gamma-ray detected sources from the CJF sample versus gamma-ray luminosity (filled symbols are gamma-ray variable while open symbols are non-variable in gamma-rays). Taken from Karouzos et al. (2011).

connected to jet speeds; iii) a correlation between gamma-ray luminosity and apparent velocity is found (higher velocity for stronger gamma-variable sources); iv) gamma-ray detected sources appear wider and with larger jet distortions. Different findings with respect to the previous studies may be due to different observing frequency (probing either different jet regions or structures) or the difference in sampling of the proper motion data.

Reference: Karouzos et al. (2011).

\subsection{F. D'Ammando - To be or not to be a blazar. The case of gamma-ray narrow line Seyfert 1 SBS 0846+513 (contribution)}

In 2008, the first NLS1 PMN J0948+0022 was detected by the Fermi-LAT. After that another four NLS1s were detected in gamma-rays. These results confirm the presence of relativistic jets also in NLS1s even though these sources are typically thought to be hosted in spiral galaxies. Their average spectral energy distributions (SEDs) are similar to FSRQs, but at lower luminosity. SBS $0846+513$ is a new gamma-ray NLS1 clearly detected during the third year of Fermi operation, in particular during a flaring state in 2011 June-July. The gamma-ray peak on daily timescale corresponds to an isotropic luminosity of about $10^{48} \mathrm{erg} \mathrm{s}^{-1}$, comparable to that of luminous FSRQs. While the kpcscale structure is unresolved in VLA images, there is a core-jet structure seen in VLBA images. The mechanism at work for producing a relativistic jet in NLS1s is not clear. Fundamental parameters should be the BH mass and the BH spin. This source could be a blazar with a BH mass at the low end of the blazar's BH mass distribution. Gamma-ray NLS1s have larger masses with respect to the entire sample of NLS1s. Moreover prolonged accretion episodes could spin-up the $\mathrm{SMBH}$ leading to a relativistic jet formation.

References: Abdo et al. (2009a), D'Ammando et al. (2012). 
OJ $287,6.2 \mathrm{~cm}$, SRT-Effelsberg.

6 April 2012, B=92 $000 \mathrm{~km}, 65 \mathrm{~s}$

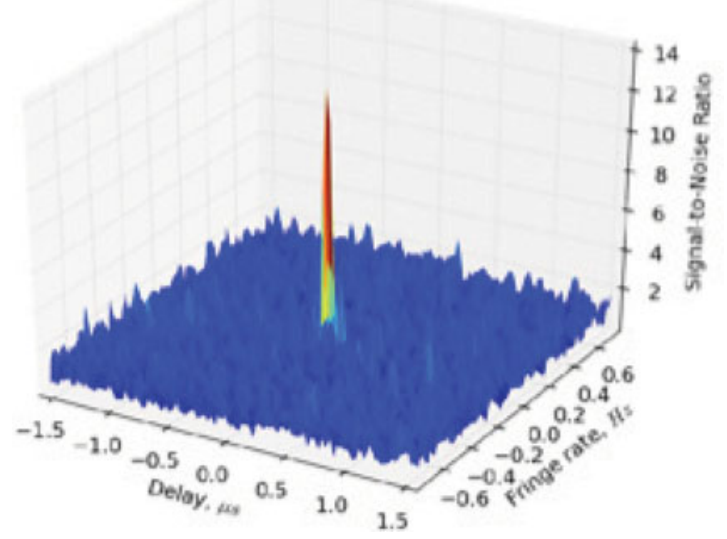

Figure 3. Early fringe obtained for OJ 287 at $6.2 \mathrm{~cm}$ between the RadioAstron space radio telescope and the ground-based Effelsberg $100 \mathrm{~m}$ with a baseline of 7.2 Earth diameters. Taken from Kardashev et al. (2013).

\section{Session 2: High resolution core and jet properties}

\subsection{N. Kardashev - RadioAstron Space VLBI mission: early results (invited)}

RadioAstron was launched on 18th July 2011. The orbital period is 8.5 days, with a perigee radius $=67,000 \mathrm{~km}$ and an apogee $=282,000 \mathrm{~km}$. Observations are carried out at P, L, C, and K bands in dual-polarization, with finest angular resolutions $(300,000$ $\mathrm{km}$ baseline) of 580, 113, 39, and 7.5-10 $\mu$-arcsec, respectively. Among the first sources detected was a single giant pulse in PSR B0950+08 at $92 \mathrm{~cm}$, with significant variations in $1 \mathrm{hr}$ due to ISM plasma scintillations. The first fringes in K-band were observed together with baselines to the Effelsberg $100 \mathrm{~m}$ from the quasar 2013+370; see Fig. 3 for another example. Imaging of the quasar $0716+714$ at $4.8 \mathrm{GHz}$ was obtained using the space radio telescope and eight ground telescopes showing the core-jet structure as seen in previous VLBI images. A status and more recent summary of the RadioAstron results appeared recently.

Reference: Kardashev et al. (2013).

\subsection{Lister - Blazars at high resolution: what large multi-epoch VLBI studies can tell us (invited)}

Blazar studies suffer heavy sample selection effects: e.g., obscuration in optical and X-ray; spectral contamination from accretion disk emission and from lobe (unbeamed) emission; non simultaneous observations; and more. To address blazar sample biases it is important to concentrate on 'uncontaminated' bands. The Fermi 2LAC AGN catalog has no contamination from the host galaxy, even if may still be incomplete due to issues with source associations. The MOJAVE VLBA program provides regular observations of radio-bright AGNs at $15 \mathrm{GHz}$. With $24 \mathrm{hrs}$ observing sessions every 3 weeks, it assures continuous time baseline data on many sources back to 1994. Among the main results we quote: the brightest gamma-ray and radio-selected quasars have similar redshift distributions; gamma-ray selected blazars have an additional sub-population of low-redshift HSP BL Lacs that are intrinsically very bright in gamma-rays; lowest luminosity BL Lacs (HSPs) all have high gamma-ray loudness (due to SED peak location). In BL Lacs (HSP and LSP) the photon index is well correlated with the Compton peak location. This trend 
could not exist if the gamma-ray and pc scale radio jet emission were fully independent. Analyzing kinematics of 889 discrete features in 201 jets from 1994 to 2011, we derive that: jets of HSP BL Lacs are characterized by lack of compact superluminal features; BL Lac jets have lower radio synchrotron luminosity and lower speeds.

References: Lister et al. (2011), Ackermann et al. (2011b).

\subsection{Orienti - On the connection between radio and gamma rays. The extraordinary case of the flaring blazar PKS 1510-089 (contribution)}

The FSRQ, PKS 1510-089 $(z=0.361)$, shows strong variability and highly superluminal jet components found close in time with gamma-ray flares. Moreover, it was detected at VHE gamma-rays, shows a high level of polarized emission, and a large rotation of the electric vector position angle (EVPA) close in time with a gamma-ray flare. PKS 1510089 underwent a very active period in 2011 reaching its historical maximum flux density in October 2011. The gamma-ray flare in July 2011 occurred after a rotation of 380 degree of the optical EVPA suggesting a common region for the optical and gamma-ray emission. The new jet component is likely evidence of a shock propagating downstream along the jet. If the gamma-ray flare in October 2011 is related to the radio outburst, it would strongly support the idea that some gamma-ray flares are produced parsecs aways from the nucleus. Note that not all flares have the same characteristics, suggesting shocks with different properties. Follow-up in the mm regime with a high sensitivity VLBI array including ALMA will be crucial in determining the high-energy emitting region.

Reference: Orienti et al. (2013).

\subsection{Z. Abraham - The radio counterparts of the 2009 exceptional gamma-ray flares in $3 C 273$ (contribution)}

A very strong and complex gamma-ray flare was observed in 3C 273 in September 2009. The flare was related to the formation of superluminal components studied at $43 \mathrm{GHz}$ and to a radio flare observed with the Itapetinga radio telescope at $43 \mathrm{GHz}$. The gammaray flux increased by a factor of 20 , while only by a factor two in radio. We explain this fact as a change in the Doppler factor, due to a change in the angle between the jet and the line of sight. This fact was predicted by the precessing jet model of Abraham \& Romero (1999). The precessing model based on the jet curvature and proper motions is compatible with a period of 16 years.

Reference: Abraham \& Romero (1999), Jorstad et al. (2011).

\subsection{Z. Shen - High precision position measurements of the cores in $3 C 66 \mathrm{~A}$ and $3 C 66 B$ (invited)}

$3 \mathrm{C} 66 \mathrm{~A}$ is a low frequency peaked BL Lac object at $z=0.444$. It is characterized by prominent variability at radio, IR, and optical frequencies. It shows a one-sided core-jet structure with detected superluminal motion. The core shift with frequency has been estimated in 2001 and 2006. A large difference has been found between the two measurements possibly due to a strong flux density increase at $15 \mathrm{GHz}$ in 2006 . This radio flare is possibly due to the core activity as shown by a new component that emerged from the central core region. Because of their small angular distance in the plane of the sky, $3 \mathrm{C} 66 \mathrm{~A}$ and the radio galaxy $3 \mathrm{C} 66 \mathrm{~B}$, are an ideal pair to obtain a combined core-shift measurement of the two sources. Comparison with data at different epochs is confusing: the difference in core shift result cannot be simply explained by the core flare activity. Other parameters apart from core flux variability may influence the core shift. More data are required to explore this point. 


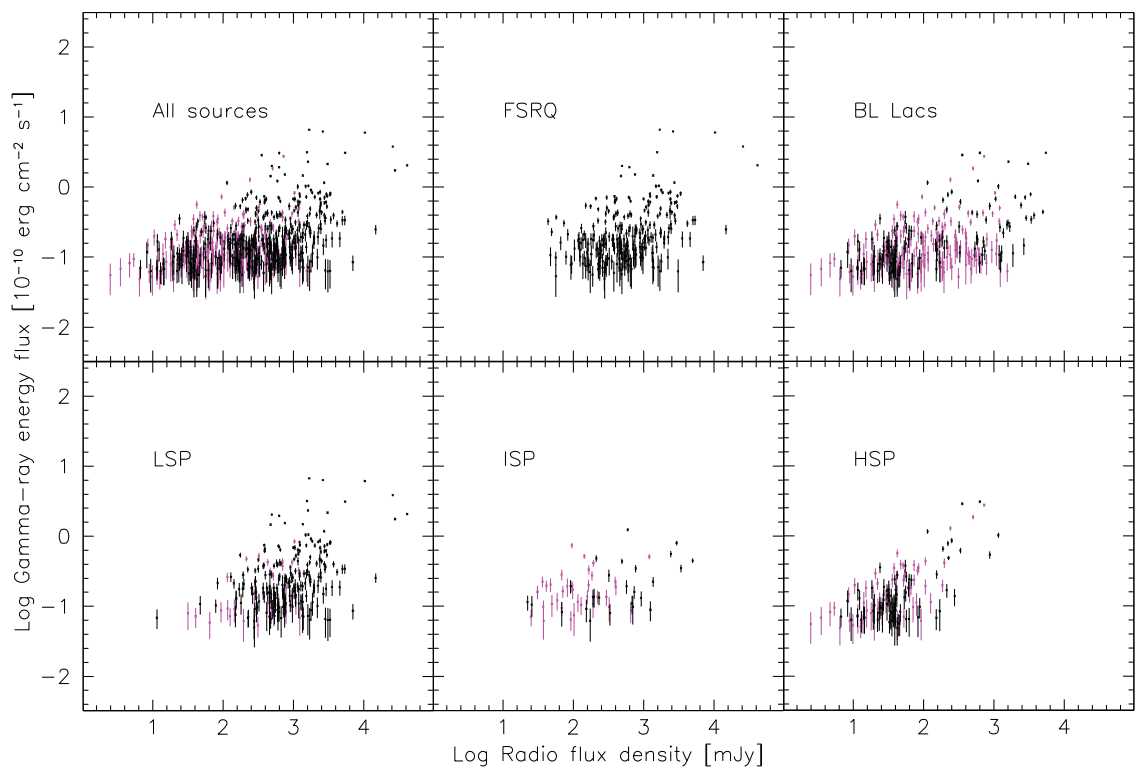

Figure 4. Broad band gamma-ray energy flux vs. $8 \mathrm{GHz}$ archival radio flux densities for the 1LAC sample separated by source type. Taken from Ackermann et al. (2011a).

References: Cai et al. (2007), Sudou \& Iguchi (2011), Zhao et al. (2011), Zhao et al. (2013).

\section{Session 3: Multi-wavelength correlations and variability}

\subsection{F. Tavecchio - Variability of blazars: probing emission regions and acceleration processes (invited)}

Blazars show variability on timescale of days suggesting a parsec scale emission region. Comparing the radio-gamma variability, there is evidence that this active region should be inside the broad line region (BLR). Moreover, in some sources the $\mathrm{GeV}$ emission shows spectral breaks that could be due to absorption effects inside the BLR. In this context, a relevant case to consider is that of PKS $1222+216(4 \mathrm{C}+21.35)$ which doubled its $\mathrm{TeV}$ flux on a timescale on the order of 10 minutes (Aleksic et al. 2011). In this source the location of the VHE emission inside the BLR is problematic because the too strong absorption expected (e.g., Liu et al. 2008, Reimer 2007, Tavecchio \& Mazin 2009, Poutanen \& Stern 2010) due to the huge optical depth of the BLR. Possibilities to reconcile rapid variability in regions at large distances from the core (outside the BLR) require the presence of jet substructure (e.g., Ghisellini \& Tavecchio 2008). Many models have been proposed but not all problems are solved - e.g., the presence of mini-jets from fast reconnection in a highly magnetized jet (Giannios et al. 2009, 2010), or narrow electron beams from magnetocentrifugal acceleration (e.g., Ghisellini et al. 2009), beams from relativistic reconnection (e.g., Cerutti et al. 2012, Nalewajko et al. 2012), or ultrahigh energy (UHE) neutral beams (Dermer et al. 2012). In conclusion, rapid variability is perhaps currently the most compelling issue in high-energy astrophysics. The idea of a unique, large, "relaxed" emission region is, at least sometimes, inadequate. 
4.2. T. Hovatta - Assessing AGN Variability and Cross-waveband Correlations in the Era of High-Quality Monitoring Data in Low and High Energies (invited)

A relevant problem in AGN is where the high-energy emission is located: close to the $\mathrm{BH}$ within the BLR or further down in the parsec-scale jet? Correlations can be used to locate the unresolved gamma-ray emission site. We can have a flux-flux correlation (amplitude domain; e.g., Fig. 4) or a light curve cross-correlation (time domain). Using simultaneous data, an intrinsic radio/gamma-ray flux density correlation is confirmed. FSRQs and BL Lacs show a different behavior (a possible selection effect?). Using archival non-contemporaneous data to increase the statistics, the correlation persists (it is even stronger for BL Lacs because of more sources).

More difficult is to tell if individual events are correlated and what are the time delays. Light curve correlations are difficult to establish in single sources. Opacity effects are important in the radio bands, moreover, we could still have too short time series. Stacked correlations show statistically significant time delays with increasing delays for longer wavelengths. Good multiwavelength coverage is really necessary to address this issue.

References: Kovalev et al. (2009), Ackermann et al. (2011a), Mahony et al. (2010), Nieppola et al. (2011), Max-Moerbeck (2012), Pavlidou et al. (2012).

\subsection{Liu - VLBI core flux density and position angle analysis of the MOJAVE AGN (contribution)}

The two-fluid jet model assumes that: 1) the outflow consists of an electronproton plasma (the jet), moving at mildly relativistic speed, 2) an electron-positron plasma (the beam) is moving at highly relativistic speed, and 3) the magnetic field lines are parallel to the flow in the beam and the mixing layer, and are toroidal in the jet.

To confirm and investigate this jet structure we model-fit the MOJAVE blazar core sample which includes blazars with more than 10 years of VLBA monitoring, and more than 15 observed epochs with a good time distribution. This sample consists of 104 sources, 77 of which are quasars, 27 BL Lacs, in which 82 are Fermi-LAT detected, and 22 non-detected sources. Of these, nine are also TeV sources. The model-fit result of the cores of 104 blazars from the MOJAVE monitoring data, suggests that Fermi LAT-detected blazars have wider position angle changes of the inner-jet than LAT nondetected blazars, and are preferentially associated with higher variable blazars.

A two-zone jet model can explain the correlations in the model-fitted parameters. The Fermi GeV gamma-ray detection rate show equally similar fraction for sources dominated by the innermost jet (zone-1) and sources dominated by the outer jet (zone-2). But importantly, $\mathrm{TeV}$ gamma-ray sources are associated mostly with blazars dominated by the outer part of inner-jet (zone-2).

Reference: Lister et al. (2009).

\subsection{E. Valtaoja - Gamma-ray emission along the radio jet: studies with Planck, Metsähovi and Fermi data (contribution)}

If the region emitting at VHE is close to the black hole - accretion disk region (i.e., it is inside the BLR), we expect that gamma-ray flares precede radio variations (assuming as VLBI zero epoch the beginning of a millimeter flare), and little or no correlation with radio variations. If the $\mathrm{VHE}$ region is distant, at or downstream of the radio core (i.e. outside the BLR), we expect gamma-ray flares simultaneous, or after, the beginning of radio variations and a correlation between $\mathrm{VHE}$ and radio variations.

Comparing data from Fermi and the Metsähovi radio sample, the case for "distant" gamma-ray origin appears much stronger. Direct observational evidence for "close" origin 


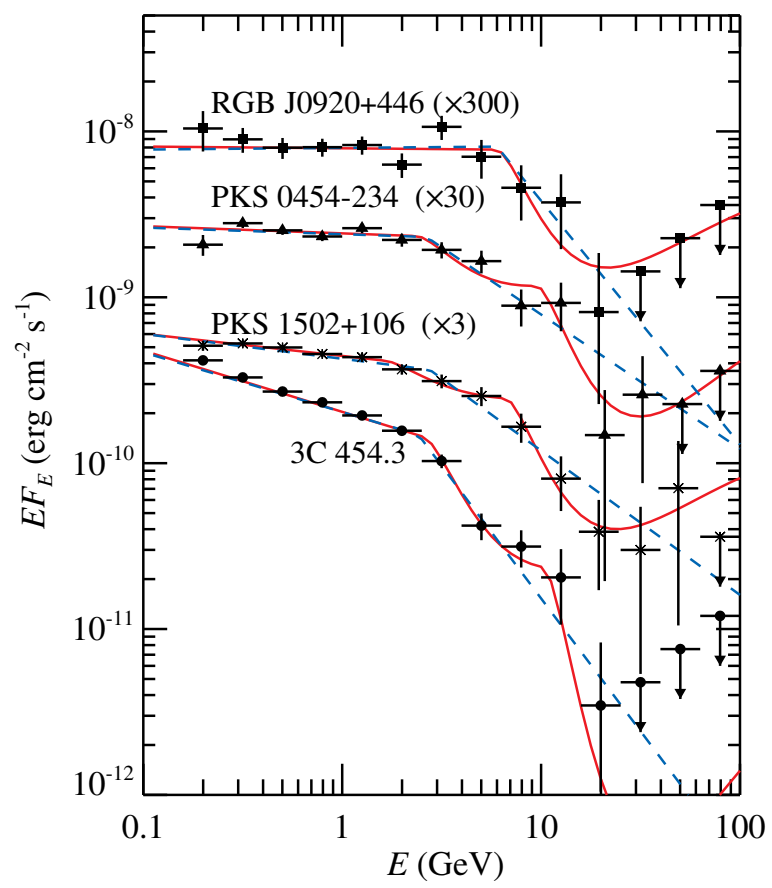

Figure 5. Fermi-LAT SEDs of four blazars with best-fit broken power law (blue dash lines) and power law with absorber model (red lines). Figure taken from Poutanen \& Stern (2010).

was not found; observations point towards distant origins: in the radio-gamma correlations, there are evident delays from radio to gamma. A confirmation of this result will be possible from the final Planck data to model the radio to gamma-ray SEDs with unprecedented accuracy.

Reference: León-Tavares et al. (2011).

\section{Session 4: Jet physics and the role of $\mathrm{BH}$ spin and $\mathrm{BH}$ accretion}

\subsection{A. Tchekhovskoy - What Sets the Power of Jets from Accreting Black Holes? (invited)}

It is crucial to understand what sets the maximum power of jets, if jets are powered by black holes (BHs) or inner regions of the accretion disks. Jet power depends on magnetic field topology: dipolar geometry gives powerful jets, quadripolar or toroidal gives weak or no jets. Jet power increases with increasing $\mathrm{BH}$ magnetic flux. BH and a large magnetic flux give a magnetically-arrested accretion (MAD): the $\mathrm{BH}$ is saturated with flux, and the $B$-field is as strong as gravity. Radio-loud AGN have MADs with BH spins $(a)$ near to 1 and radio-quiet AGN shows MADs with $a<0.1$. Retrograde BHs appear to have less powerful jets while thicker disks show more powerful jets.

References: Tchekhovskoy et al. (2010), Tchekhovskoy et al. (2011), McKinney et al. (2012), Tchekhovskoy \& McKinney (2012).

\subsection{J. Poutanen - Fermi observations of blazars: implications for gamma-ray production (contribution)}

The presence in the blazar spectra of $\mathrm{GeV}$ breaks (see Fig. 5 for examples) produced by photon-photon absorption on BLR photons proves that gamma-rays are produced 
within the BLR. These spectral breaks are relatively stable during flares. Most GeV breaks cannot be produced by Ly- $\alpha$ photons, but can be produced by Lyman continuum of ionized helium. The gamma-ray emitting region is located at the boundary of the $\mathrm{He}^{++}$zone and moves away from the BLR high-ionization zone (moving region model). This model is consistent with the arrival of photons with energy larger than $10 \mathrm{GeV}$ in the end of the flare. Alternative interpretations that gamma-rays are produced together or even after radio flares are ambiguous.

References: Abdo et al. (2010b), Abdo et al. (2011), Poutanen \& Stern (2010), Stern \& Poutanen (2011).

\subsection{S. Trippe - Probing the $\mathrm{mm} /$ radio polarization of active galactic nuclei (contribution)}

The Plateau de Bure Interferometer (PdBI) is located in the south-east of France, consists of six 15-m antennas, and observes with dual-polarization single-band receivers in the frequency range $80371 \mathrm{GHz}$, with a maximum baseline of $760 \mathrm{~m}\left(\sim 0.2^{\prime \prime}-7^{\prime \prime}\right.$ angular resolution). Each PdBI observation collects polarization information on calibration quasars (since 2007), thus resulting in a large mm polarization survey at $1.3 / 2 / 3$ $\mathrm{mm}$ "for free." Four years of polarization monitoring data of AGN were used for this study. Polarization is detected in almost all sources (73 out of 86 quasars), consisting of 316 detections out of 441 source measurements. Fast variability in flux and polarization are observed with almost identical fluctuation rates, implying similar spatial scales are probed. Polarization variations indicate strong shocks and allow for estimates of the shock parameters. Very large rotation measures (up to 400,000 radians $\mathrm{m}^{-2}$ ) are measured and allow an estimate of the outflow geometries (spherical/conical outflows).

References: Trippe et al. (2010), Trippe et al. (2012).

\subsection{Su-Fermi Gamma-Ray bubbles, jets, and lines in the Milky Way (contribution)}

The Fermi bubbles are giant gamma-ray structures with sharp edges discovered using data from the Fermi-LAT. They rise up \& down from the Galactic center with extents of $\sim 50$ degrees $(\sim 8.5 \mathrm{kpc})$, are well centered on longitude zero and close to latitude zero, and imply the acceleration of $\mathrm{TeV}$ electron energy particles. They could be related to a jet or outflow activity from the Galactic center. The gamma-ray bubbles have counterparts at microwave frequencies (the WMAP haze, confirmed by Planck). Sharp edges are observed in X-ray also utilizing ROSAT data, and several small XMM-Newton pointings have recently been obtained in order to study this in more detail. The basic question surrounding the origin of the bubbles is whether they are jet or wind/outflow driven. A gamma-ray jet / cocoon feature was recently detected in the Fermi-LAT data offering additional clues. Overall, the Fermi bubbles are analogous to large-scale structures observed in more distant AGN, e.g., Cen A, and connections are being sought.

References: Su et al. (2010), Su \& Finkbeiner (2012).

\section{Acknowledgements}

We would like to acknowledge the support from the IAU, the JD6 participants for their contributions, and members of the organizing committee: Ed Fomalont, Luigi Foschini, Marcello Giroletti, Xiaoyu Hong (co-chair), Seiji Kameno, Matthias Kadler, Yuri Kovalev, Laura Maraschi (co-chair), David Paneque, Maria Rioja, Eduardo Ros, Łukasz Stawarz, Meg Urry, and Anton Zensus. T.C. was supported at NRL by NASA DPR S-15633-Y. 


\section{References}

Abdo, A. A., Ackermann, M., Ajello, M., et al. 2009a, ApJ, 699, 976 (PMN J0948+0022)

Abdo, A. A., Ackermann, M., Ajello, M., et al. 2009b, ApJL, 707, L142 (LAT radio-loud NLS1s)

Abdo, A. A., Ackermann, M., Ajello, M., et al. 2010a, Science, 328, 725 (Cen A lobes)

Abdo, A. A., Ackermann, M., Ajello, M., et al. 2010b, ApJ, 710, 1271 (LAT blazar spectra)

Abdo, A. A., Ackermann, M., Ajello, M., et al. 2011, ApJL, 733, L26 (3C 454.3 flare)

Abraham, Z. \& Romero, G. E. 1999, A\& A, 344, 61

Abramowski, A., Acero, F., Aharonian, F., et al. 2012, ApJ, 746, 151 (M87)

Acciari, V. A., Aliu, E., Arlen, T., et al. 2011, ApJ, 738, 25 (Mrk 421)

Ackermann, M., Ajello, M., Allafort, A., et al. 2011a, ApJ, 741, 30 (radio-gamma connection)

Ackermann, M., Ajello, M., Allafort, A., et al. 2011b, ApJ, 743, 171 (2LAC)

Ackermann, M., Ajello, M., Allafort, A., et al. 2012, ApJ, 747, 104 (LAT Seyferts)

Aleksić, J., Alvarez, E. A., Antonelli, L. A., et al. 2012, A\&\&A, 542, A100 (Mrk 421)

Cai, H.-B., Shen, Z.-Q., Sudou, H., et al. 2007, A\&AA, 468, 963

Cerutti, B., Werner, G. R., Uzdensky, D. A., \& Begelman, M. C. 2012, ApJL, 754, L33

D'Ammando, F., Orienti, M., Finke, J., et al. 2012, MNRAS, 426, 317

Dermer, C. D., Murase, K., \& Takami, H. 2012, ApJ, 755, 147

Ghirlanda, G., Ghisellini, G., Tavecchio, F., Foschini, L., \& Bonnoli, G. 2011, MNRAS, 413, 852

Ghisellini, G. \& Tavecchio, F. 2008, MNRAS, 386, L28

Ghisellini, G., Tavecchio, F., Bodo, G., \& Celotti, A. 2009, MNRAS, 393, L16

Giannios, D., Uzdensky, D. A., \& Begelman, M. C. 2009, MNRAS, 395, L29

Giannios, D., Uzdensky, D. A., \& Begelman, M. C. 2010, MNRAS, 402, 1649

Hayashida, M., Madejski, G. M., Nalewajko, K., et al. 2012, ApJ, 754, 114

HESS Collaboration: Abramowski, A., Acero, F., et al. 2012, A\&AA, 545, A103

Jorstad, S., Marscher, A., Agudo, I., \& Harrison, B. 2011, 2011 Fermi Symposium, eConf C110509, arXiv:1111.0110

Kardashev, N. S., Khartov, V. V., Abramov, V. V., et al. 2013, Astronomy Reports, 57, 153

Karouzos, M., Britzen, S., Witzel, A., Zensus, J. A., \& Eckart, A. 2011, AESA, 529, A16

Kovalev, Y. Y., Aller, H. D., Aller, M. F., et al. 2009, ApJL, 696, L17

León-Tavares, J., Valtaoja, E., Tornikoski, M., Lähteenmäki, A., \& Nieppola, E. 2011, AE\&A, 532, A146

Lister, M. L., Cohen, M. H., Homan, D. C., et al. 2009, AJ, 138, 1874

Lister, M. L., Aller, M., Aller, H., et al. 2011, ApJ, 742, 27

Liu, H. T., Bai, J. M., \& Ma, L. 2008, ApJ, 688, 148

Mahony, E. K., Sadler, E. M., Murphy, T., et al. 2010, ApJ, 718, 587

Max-Moerbeck, W. 2012, BAAS, 219, \#321.05

McConnell, D., Sadler, E. M., Murphy, T., \& Ekers, R. D. 2012, MNRAS, 422, 1527

McConville, W., Ostorero, L., Moderski, R., et al. 2011, ApJ, 738, 148

McKinney, J. C., Tchekhovskoy, A., \& Blandford, R. D. 2012, MNRAS, 423, 3083

Nalewajko, K., Begelman, M. C., Cerutti, B., Uzdensky, D. A., \& Sikora, M. 2012, MNRAS, 425,2519

Nieppola, E., Tornikoski, M., Valtaoja, E., et al. 2011, A\& A, 535, A69

Orienti, M., Koyama, S., D'Ammando, F., et al. 2013, MNRAS, 428, 2418

Paneque, D., Ballet, J., Burnett, T., et al. 2012, 4th Fermi Symposium, eConf C121028, arXiv:1304.4153

Pavlidou, V., Richards, J. L., Max-Moerbeck, W., et al. 2012, ApJ, 751, 149

Poutanen, J. \& Stern, B. 2010, ApJL, 717, L118

Reimer, A. 2007, ApJ, 665, 1023

Sudou, H. \& Iguchi, S. 2011, AJ, 142, 49

Stern, B. E. \& Poutanen, J. 2011, MNRAS, 417, L11

$\mathrm{Su}$, M., Slatyer, T. R., \& Finkbeiner, D. P. 2010, ApJ, 724, 1044

Su, M. \& Finkbeiner, D. P. 2012, ApJ, 753, 61

Takeuchi, Y., Kataoka, J., Stawarz, Ł., et al. 2012, ApJ, 749, 66

Tavecchio, F. \& Mazin, D. 2009, MNRAS, 392, L40 
Tchekhovskoy, A., Narayan, R., \& McKinney, J. C. 2010, ApJ, 711, 50

Tchekhovskoy, A., Narayan, R., \& McKinney, J. C. 2011, MNRAS, 418, L79

Tchekhovskoy, A. \& McKinney, J. C. 2012, MNRAS, 423, L55

Trippe, S., Neri, R., Krips, M., et al. 2010, A\&\&A, 515, A40

Trippe, S., Neri, R., Krips, M., et al. 2012, A\& A, 540, A74

Zhao, G.-Y., Chen, Y.-J., Shen, Z.-Q., et al. 2011, Journal of Astrophysics 83 Astronomy, 32, 61

Zhao, G.-Y., Chen, Y.-J., Shen, Z.-Q., et al. 2013, IAU Symposium, 290, 367 